\title{
Patient characteristics, diagnosis, and treatment of non-contact lens related Acanthamoeba keratitis
}

\author{
Savitri Sharma, Prashant Garg, Gullapalli N Rao
}

\begin{abstract}
Aim-To review the clinical characteristics, diagnosis, and visual outcome in patients with non-contact lens related Acanthamoeba keratitis and compare the findings with reported series of contact lens associated Acanthamoeba keratitis. Methods-Medical and microbiology records of 39 consecutive patients with a diagnosis of Acanthamoeba keratitis, at a tertiary eyecare centre in India between January 1996 and June 1998, were analysed retrospectively.

Results-A majority of the patients presented with poor visual acuity and large corneal stromal infiltrates (mean size 38.20 (SD 26.18) $\mathrm{mm}$ ). A predisposing factor was elicited in $19 / 39(48.7 \%)$ patients (trauma 15, dirty water splash three, leaf juice one). None of the patients had worn contact lenses. Most patients (26/39 $(66.6 \%)$ ) came from a low socioeconomic background. Complaint of severe pain was not a significant feature and radial keratoneuritis was seen in $1 / 39(2.5 \%)$ patients. A ring infiltrate was present in $41.1 \%$ of cases. A clinical diagnosis of fungal keratitis was made in $45 \%$ of the patients before they were seen by us. However, all patients were diagnosed microbiologically at our institute based on demonstration of Acanthamoeba cysts in corneal scrapings (34/ 39) and/or culture of Acanthamoeba (34/ 39). Treatment with biguanides (PHMB, 15/38 (39.4\%), PHMB with CHx, 23/38 $(60.5 \%)$, one patient did not return for treatment) resulted in healing with scar formation in 27 out of $31(87.0 \%)$ followed up patients (mean time to healing 106.9 days). Overall visual outcome was poor with no statistical difference between cases diagnosed within $\mathbf{3 0}$ days (early) or $\mathbf{3 0}$ days after (late) start of symptoms. The visual outcome in cases requiring tissue adhesive (five) and keratoplasty (three) was also poor.

Conclusions-This is thought to be the largest series of cases of Acanthamoeba keratitis in non-contact lens wearers. In such cases, the disease is advanced at presentation in most patients, pathognomonic clinical features are often not seen, disease progression is rapid, and visual outcome is usually poor. Possible existence of Acanthamoeba pathotypes specifically associated with non-contact lens keratitis and unique to certain geographical areas is suggested.

(Br F Ophthalmol 2000;84:1103-1108)
\end{abstract}

From a historical perspective Acanthamoeba keratitis has been described as a recent epidemic. ${ }^{1}$ It has been recognised in almost all parts of the world. ${ }^{2-6}$ Evidence from several studies has suggested soft contact lens wear as the greatest risk factor ${ }^{7} 8$ although some studies have reported the development of Acanthamoeba keratitis in patients with no apparent predisposing factor. ${ }^{4}$ With most of the literature focusing on contact lens related Acanthamoeba keratitis, ophthalmologists may hesitate to diagnose this entity in patients without lenses. Chynn et $a l^{10}$ reported some of the differences between contact lens and noncontact lens related Acanthamoeba keratitis in a small number of patients. Mean time to diagnosis was significantly longer and visual outcome was significantly lower in non-contact lens users compared with those who wore contact lenses. The study recommended the need for increased suspicion of Acanthamoeba keratitis in patients who did not present with the obvious risk factor of contact lenses. On the basis of our experience, on a much larger scale, with Acanthamoeba keratitis unrelated to contact lens wear, we support the above contention and report in this study 39 cases of Acanthamoeba keratitis in non-contact lens users. We present the clinical characteristics, diagnostic methods, treatment schedules, and outcome in these cases and discuss our findings in the context of reported series of Acanthamoeba keratitis in patients using contact lenses.

\section{Patients and methods}

All patients treated for Acanthamoeba keratitis between January 1996 and June 1998 were included in the study. The clinical and microbiology records were reviewed retrospectively. Information obtained from the clinical records included age, sex, socioeconomic status, medical history including predisposing risk factors, duration and type of symptoms, prior diagnosis and treatment, presenting ocular findings, ocular treatment, and final visual outcome.

Laboratory data of all patients were sought from microbiology records. Results of smear examinations of corneal scrapings which included Gram stain, Giemsa stain, and potassium hydroxide with calcofluor white $(\mathrm{KOH}+\mathrm{CFW})$ stain were noted. Culture results indicating any significant growth of bacteria, fungus, or Acanthamoeba were recorded along with the in vitro sensitivity test results. Cultures in all cases had included a variety of media, such as sheep blood agar (aerobic, anaerobic), chocolate agar, brain-heart infusion broth, thioglycollate broth, Sabouraud's 
Table 1 Age and sex distribution of patients with laboratory confirmed Acanthamoeba keratitis

\begin{tabular}{llll}
\hline & \multicolumn{2}{c}{ Category } & \\
\cline { 2 - 3 } Patients & Early & Late & Total \\
\hline Sex & & & \\
$\quad$ Male & 15 & 8 & 23 \\
$\quad$ Female & 9 & 7 & 16 \\
Age (years) & 1 & 0 & 1 \\
$0-20$ & 14 & 11 & 25 \\
$21-40$ & 8 & 4 & 12 \\
$41-60$ & 1 & 0 & 1 \\
$\geqslant 61$ & 36.75 & 37.00 & 36.8 \\
Mean age (years) & 24 & 15 & 39 \\
No of patients & & \\
\hline
\end{tabular}

Early $=$ cases diagnosed within 30 days of start of symptoms.

Late $=$ cases diagnosed 30 days after start of symptoms.

dextrose agar, and non-nutrient agar with Escherichia coli overlay, allowing for the growth of bacteria, fungus, or Acanthamoeba. Significance was assigned to the bacterial or fungal growth if the same organism had grown in more than one medium or a similar organism was seen in the smears of corneal scrapings. All organisms including Acanthamoeba were identified using standard procedures. ${ }^{11}$

Once the diagnosis was established by microbiological investigation the treatment was started with polyhexamethylene biguanide $0.02 \%$ (PHMB, Baquasil, ICI, USA) and/or chlorhexidine digluconate $0.02 \% \quad(\mathrm{CHx}$, Sigma, C-9394) instilled half hourly to the affected eye day and night for 2-3 days (usually as an inpatient) and then 1 hourly for a week. The frequency of instillation of the biguanides was reduced to 3 or 4 hourly per day according to clinical response over subsequent weeks and continued after resolution of inflammatory signs for 2-3 weeks. In $18(46.1 \%)$ cases Neosporin ointment (neomycin, Dominion) was added at night. Concomitant bacterial infection was treated with ciprofloxacin $0.3 \%$ eye drops and modified if required according to the sensitivity results from the laboratory. Topical cycloplegics and oral analgesics were used in most cases. Steroids were not used in any case.

For analysis, the patients were divided into two groups according to the time between the onset of symptoms and diagnosis of Acanthamoeba keratitis (initiation of antiamoebic treatment). The "early" category consisted of patients who were diagnosed within 1 month of onset of symptoms and the remaining were labelled as "late".

\section{Results}

Over a span of $2^{1 / 2}$ years, we treated 39 eyes of 39 patients with Acanthamoeba keratitis. Table 1 shows the demographic data of these patients. The laboratory data are listed in Table 2.

More males than females (23:16) were affected, though the difference was not significant $(\mathrm{p}=0.2945)$. Mean age of patients was 36.8 years with a range of $18-70$ years.

All except six patients had received ophthalmic attention before reporting to our cornea service and were being treated for presumed bacterial, fungal, or viral keratitis based on clinical features. None had been investigated
Table 2 Laboratory data of early and late cases of Acanthamoeba keratitis

\begin{tabular}{|c|c|c|c|}
\hline \multirow[b]{2}{*}{ Tests } & \multicolumn{2}{|l|}{ Category } & \multirow[b]{2}{*}{ Total (39) } \\
\hline & $\begin{array}{l}\text { Early cases } \\
(n=24)\end{array}$ & $\begin{array}{l}\text { Late cases } \\
(n=15)\end{array}$ & \\
\hline \multicolumn{4}{|l|}{ Acanthamoeba } \\
\hline \multicolumn{4}{|l|}{ Cysts in smears } \\
\hline Gram & $15(62.5 \%)$ & $11(73.3 \%)$ & $26(66.6 \%)$ \\
\hline Giemsa & $14(58.3 \%)$ & $9(60.0 \%)$ & $23(58.9 \%)$ \\
\hline $\mathrm{KOH}+\mathrm{CFW}$ & $21(87.5 \%)$ & $13(86.6 \%)$ & $34(87.1 \%)^{\star}$ \\
\hline \multicolumn{4}{|l|}{ Culture } \\
\hline Acanthamoeba & $21(87.5 \%)$ & $13(86.6 \%)$ & $34(87.1 \%) \dagger$ \\
\hline $\begin{array}{l}\text { Gram +ve } \\
\text { bacteria }\end{array}$ & $5(20.8 \%)$ & $4(26.6 \%)$ & $9(23.0 \%)$ \\
\hline
\end{tabular}

${ }^{\star}$ Smear -ve culture +ve - (5).

+Smear +ve culture -ve - (5)

Early $=$ cases diagnosed within 30 days of start of symptoms.

Late $=$ cases diagnosed 30 days after start of symptoms .

microbiologically. The most common clinical suspicion was of fungal keratitis (15/33, $45.4 \%$ ). Viral keratitis was suspected in only four cases. One patient was clinically suspected to have Acanthamoeba keratitis and was receiving PHMB.

None of the patients in our series wore contact lenses. While 15 patients gave a history of definite trauma with either vegetative matter, stone, or dust, three patients reported splashing unclean water into the eyes along with trauma. In one patient, a suspected foreign body on the cornea was removed with the tongue. The same patient used extract of some herbs in the eye before seeing a doctor who treated her for bacterial keratitis. In 20 $(51.2 \%)$ patients no definite risk factor could be elicited from the history.

\section{SIGNS AND SYMPTOMS}

All patients presented to us with redness, watering, and decrease in vision. Ocular pain disproportionate to the degree of keratitis was not noted for any of the patients. Various clinical signs noted in our patients are shown in Table 3. Radial keratoneuritis, which has been described as specific for the diagnosis of Acanthamoeba keratitis, was seen in only one patient. Anterior stromal infiltrates were present in all patients except one in the late group who had a corneal scar with oedema. A ring infiltrate was present in $41.1 \%$ of the patients and was the presenting feature more often $(45.8 \%)$ in the early than the late group $(33.3 \%)$. The difference was not statistically significant $(p=0.6617)$. Less specific signs

Table 3 Clinical signs in early and late cases of Acanthamoeba keratitis at presentation

\begin{tabular}{|c|c|c|c|}
\hline \multirow[b]{2}{*}{ Clinical signs } & \multicolumn{2}{|l|}{ Category } & \multirow[b]{2}{*}{$\begin{array}{l}\text { Total } \\
(n=39)\end{array}$} \\
\hline & $\begin{array}{l}\text { Early cases } \\
(n=24)\end{array}$ & $\begin{array}{l}\text { Late cases } \\
(n=15)\end{array}$ & \\
\hline Ring infiltrate & $11(45.8 \%)^{\star}$ & $5(33.3 \%)$ & $16(41.0 \%)$ \\
\hline Satellite infiltrate & $9(37.5 \%)$ & $2(13.3 \%)$ & $11(28.2 \%)$ \\
\hline Diffuse infiltrate & $11(45.8 \%)$ & $10(66.6 \%)$ & $21(53.8 \%)$ \\
\hline Endothelial plaque & $4(16.6 \%)$ & $1(6.6 \%)$ & $5(12.8 \%)$ \\
\hline Hypopyon & $14(58.3 \%)$ & $7(46.6 \%)$ & $21(53.8 \%)$ \\
\hline Dendritic lesion & $1(4.1 \%)$ & 0 & $1(2.5 \%)$ \\
\hline $\begin{array}{l}\text { Radial } \\
\text { keratoneuritis }\end{array}$ & 0 & $1(6.6 \%)$ & $1(2.5 \%)$ \\
\hline
\end{tabular}

${ }^{\star}$ In two cases the ring infiltrate was partial.

Early $=$ cases diagnosed within 30 days of start of symptoms. Late $=$ cases diagnosed 30 days after start of symptoms. 


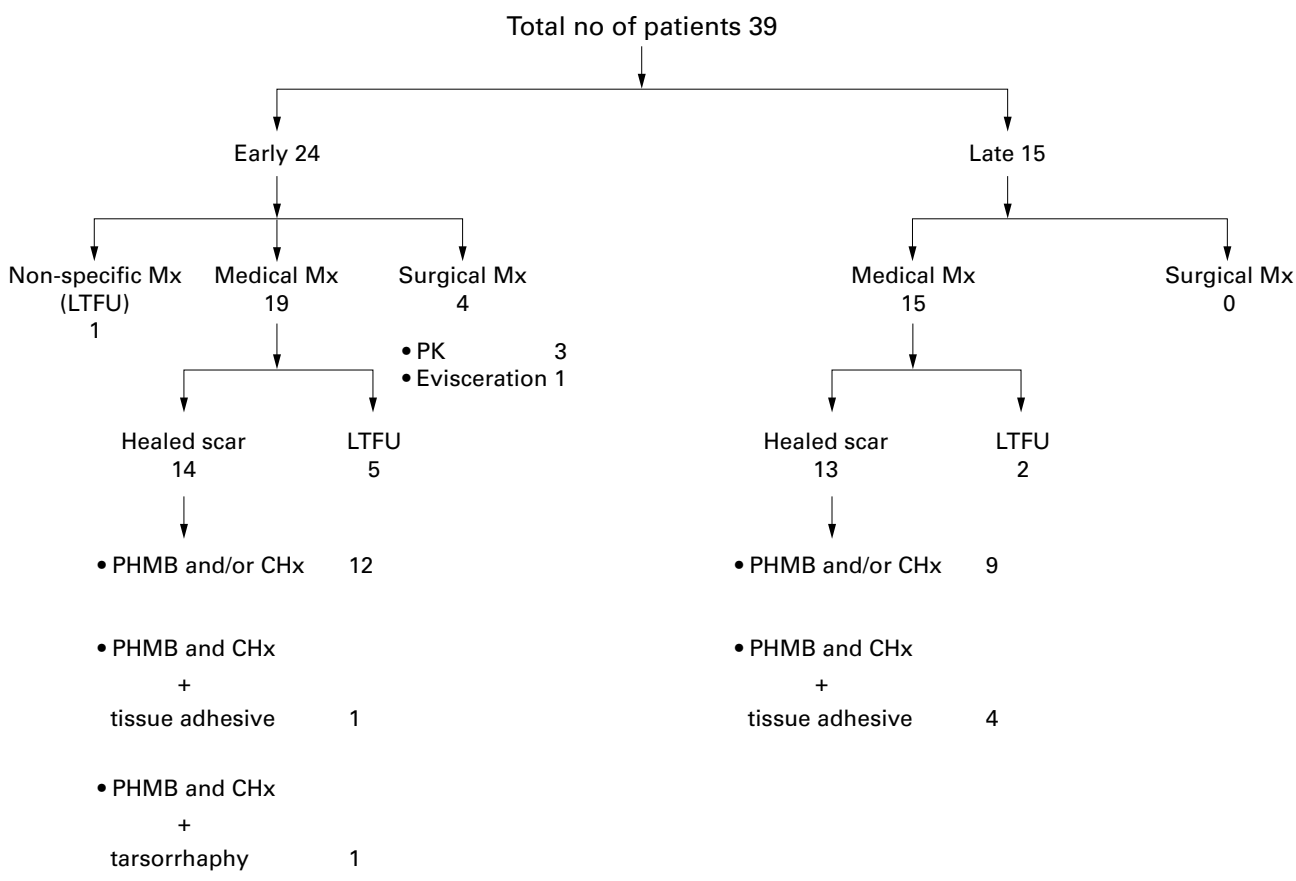

Figure 1 Therapeutic strategy and treatment outcome in 39 patients with non-contact lens related Acanthamoeba keratitis.

such as satellite stromal infiltrates, diffuse stromal infiltrates, and endothelial plaques were seen in many of the patients with no significant difference between the early and late groups. One patient in the early group presented with dendritiform epithelial lesion mimicking viral keratitis. More than half of the patients $(53.8 \%)$ had anterior chamber reaction with hypopyon ranging from trace to 3.5 $\mathrm{mm}$.

\section{DIAGNOSIS}

Diagnosis in all cases was based on the demonstration of Acanthamoeba cysts or trophozoites in corneal scrapings by smear examination and/or culture. Twenty four of 39 (61.5\%) patients were diagnosed within a month (early) and $15(38.5 \%)$ cases were diagnosed 1 month (late) after start of symptoms. Twenty nine $(74.3 \%)$ cases were positive for Acanthamoeba both on smear examination and culture where as five each were detected either by culture or smear (Table 2). Among 34 smear positive cases, $\mathrm{KOH}+\mathrm{CFW}$ stain detected cysts in all cases. Fewer cases showed cysts in corneal scrapings by Gram and Giemsa stain (26/34 $(76.4 \%)$ and $23 / 34(67.6 \%)$ respectively). Bacterial growth along with Acanthamoeba was seen in five cases in the early group and four cases in late group. The bacterial co-isolates were mainly low virulence organisms such as Staphylococcus epidermidis (6/9) and Corynebacterium species (2/9). Staphylococcus aureus was isolated in one case.

TREATMENT OUTCOME

Anti-Acanthamoeba treatment with cationic antiseptics (PHMB and/or CHx) was initiated in all cases as soon as the microbiological diagnosis was made. The therapeutic strategy and outcome in all patients is shown in Figure
1. In 27 out of 31 patients followed up $(87.0 \%)$ the corneal infiltrate resolved following medical treatment in a mean time of 106.9 (9-281) days. Seven patients were lost to follow up, four of whom were refused admission for financial reasons, and three patients left against medical advice after being treated for 5-7 days. Post-treatment visual acuity was recorded in 14 early and 13 late cases. Visual acuity before and after treatment in early and late cases is shown in Figure 2. Improvement in visual acuity following treatment was seen in $35.7 \%(5 / 14)$ of early and $30.7 \%(4 / 13)$ of late cases $(p=0.891)$. Application of tissue adhesive (n-butyl cynoacrylate tissue adhesive) with bandage contact lens was performed in five cases (four in late, one in early) for extreme thinning or perforation less than $2 \mathrm{~mm}$ in size. After prolonged medical therapy with $\mathrm{PHMB}$ and $\mathrm{CHx}$ (mean 88 days) the infiltrates resolved with scarring and

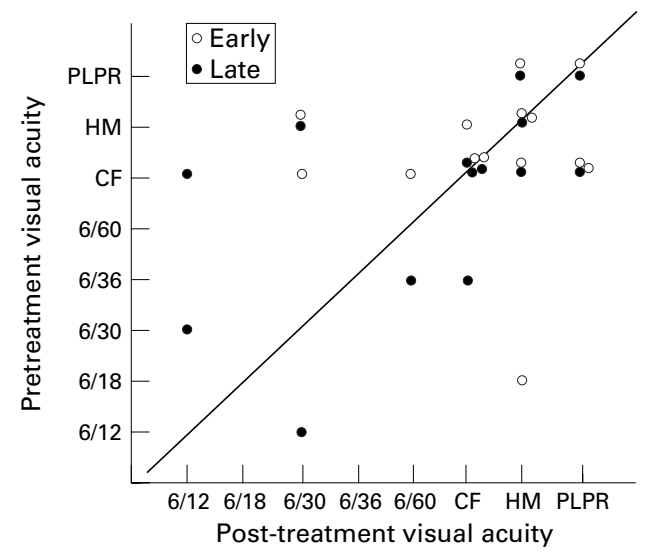

Figure 2 Pretreatment and post-treatment visual acuity in non-contact lens related Acanthamoeba keratitis patients diagnosed within 30 days (early, 14 cases) and 30 days after (late, 13 cases) start of symptoms. 
vascularisation in all. However, the final visual acuity remained poor (PL/PR three, HM one, and $6 / 36$ one). Paramedian tarsorrhaphy was performed for associated lagophthalmos in one patient. Therapeutic penetrating keratoplasty (PK) was performed in three cases in the early group owing to large infiltrate at the time of presentation which failed to respond even on intensive medical therapy. The outcome of surgical excision however, was not encouraging. One patient required limbus to limbus graft 7 days after presentation. The graft developed an infiltrate in the immediate postoperative period followed by corneal perforation and pseudocornea formation. The graft failed in the second patient, and in the third patient there was choroidal detachment with vitreous opacities resulting ultimately in phthisis. Development of vitreous opacities and scleral involvement was noted in one patient who ultimately required evisceration.

\section{Discussion}

Corneal ulceration is a major cause of monocular blindness in developing countries. A recent report from this centre, in a well conducted survey of an urban population in south India, listed corneal blindness as the third major cause of visual disability and blindness. ${ }^{12}$ Incidence of corneal ulcers in south India has been estimated to be 11.3 per 10000 population and has been referred to as a blinding disease of epidemic proportions. ${ }^{13}$ Epidemiology and aetiology of corneal ulceration in south India has already been published $^{14}$ wherein $1 \%$ of culture positive cases were caused by Acanthamoeba. The reported incidence of Acanthamoeba keratitis in India varies from $1-3 \%$ in various published $^{1415}$ and unpublished series. Of note, barring single case reports in contact lens wearers, ${ }^{16}{ }^{17}$ all reported cases are predominantly seen in non-contact lens wearers. ${ }^{414} 15$ While Sharma et $a l^{4}$ reported complete healing in four out of five adequately followed up cases (treated with neosporin/miconazole/ ketoconazole) they did not comment on visual outcome. Likewise, none of the other studies $^{14}{ }^{15}$ has dwelt on clinical features, treatment schedule, or visual outcome. Consequently, such data are severely lacking from this part of the world.

On the other hand, some reports from the $\mathrm{USA}^{10}$ and the UK ${ }^{18}$ have described the disease in non-contact lens wearers. While contributing significantly to the understanding of Acanthamoeba keratitis in non-contact lens wearers, both these studies were encumbered by relatively small sample size. To the best of our knowledge, the present study is the largest series of patients with non-contact lens related Acanthamoeba keratitis.

Diagnosis of Acanthamoeba keratitis, based on clinical features, was made only in one out of 33 patients by physicians who had treated these patients before they were seen by us. The majority of these patients $(15 / 33(45.4 \%))$ were treated as fungal keratitis which is different from what has been reported for patients with contact lens related Acanthamoeba keratitis who generally undergo treatment for herpes simplex keratitis before the microbiological diagnosis is made. ${ }^{19}$ The presence of satellite stromal infiltrates and endothelial exudates in some of our patients points more towards fungal aetiology. Additional reason for underdiagnosis of Acanthamoeba keratitis could have been the relatively high incidence of fungal keratitis in India $(50 \%),{ }^{14}$ and lack of enough reports of Acanthamoeba keratitis in noncontact lens wearers. Radial keratoneuritis, described as typical for Acanthamoeba keratitis, was also uncommon in our patients. Visual loss in contrast with severe pain was the predominant symptom in our series. Absence of complaints of pain disproportionate to clinical features in our patients is probably related to absence of keratoneuritis. Although predilection of Acanthamoeba to neural tissue has been demonstrated, ${ }^{20}$ it is possible that the same is not seen with all species and pathotypes of Acanthamoeba.

Contact lens wear does not emerge as an important risk factor for Acanthamoeba keratitis in our population. This can probably be attributed to the relatively few people who are exposed to contact lens wear in this country. In $20 / 39(51.2 \%)$ of the patients in this series a definite risk factor that led to Acanthamoeba keratitis could not be elicited. In previously published reports of this entity in non-contact lens wearers from India ${ }^{4}$ and elsewhere, ${ }^{10} 18$ trauma and exposure to contaminated water have been identified as major risk factors. Use of traditional eye medicine may be added as another risk factor though this was reported in just one patient in this series. A recent study from south India reported use of traditional eye medicine by $47.7 \%$ of patients with keratitis in a rural setting. ${ }^{21}$ However, the lone patient with Acanthamoeba keratitis in their series had not used any traditional medicine. It is possible that some of our patients experienced events that were too trivial to remember. The low socioeconomic background of our patients $(26 / 39(66.6 \%))$ may be a contributory factor. The innocuous habit of splashing water into the eye following dust fall may not be remembered by the patients and cannot be ruled out in our patients. On the other hand, it is also possible that particularly virulent strains of Acanthamoeba adhere to and invade normal corneal epithelium, a possibility suggested by the electron microscopy study of Neiderkorn et $a l^{22}$ which showed rigid host specificity of $A$ castellanii to intact chinese hamster, pig, and human corneas in vitro.

In this series the diagnosis and initiation of treatment was delayed in $38.5 \%$ of patients by more than 30 days. This is in contrast with contact lens related Acanthamoeba keratitis. Radford et $a l^{18}$ reported that diagnosis was established within 1 month in $89 \%$ of contact lens related cases. This delayed diagnosis could have been because of lack of severe pain and low socioeconomic status of most of our patients. Compared with Acanthamoeba keratitis in contact lens wearers, severe corneal features were noted in our patients at presentation. The stromal infiltrates, even in early 
cases, varied in size from $11.9-88 \mathrm{~mm}$ (mean 38.3 (SD 20.6) $\mathrm{mm}$ ). Large ulcer size and central location in $22 / 24(91.6 \%)$ patients in the early group and $13 / 15(86.6 \%)$ in late group account for the overall poor visual outcome in our patients. Though we found the final visual outcome better in cases diagnosed early than late, the difference was not signifcant $(p=0.891)$, which is in contrast to other studies which have shown a significant difference. ${ }^{10} 18{ }^{23}$ While Radford et al ${ }^{18}$ related the less assured visual outcome in patients with no contact lens history to delayed diagnosis we believe that this is also related to the presence of more severe disease in these patients. Wide variability in virulence among the strains of Acanthamoeba in different geographical areas, as well as differences in host immune responses, may contribute to the variability in clinical presentation. In the patients in this series, a rapid progression of the corneal pathology leading to early and severe damage to corneal tissues is seen. This is in contrast with slow progress of the disease in contact lens related keratitis where a marked stromal involvement may not be obvious until 6 weeks or more. ${ }^{24}$ Though the host factors may have an important part to play, variability among different strains can not be ruled out. Co-infection with bacteria may be another explanation in this setting; however, in this series there were only nine $(23 \%)$ cases with mixed infection with bacteria.

Microbiological diagnosis in this series was satisfactory in majority of the patients with the organisms being demonstrated in smears and cultures. In 32/34 cases the first scraping yielded a positive result leaving only two requiring rescrapes. As reported earlier, ${ }^{25}$ $\mathrm{KOH}+\mathrm{CFW}$ staining of corneal scrapings proved to be the most rewarding of all methods for the diagnosis of Acanthamoeba keratitis. With increased awareness and better diagnostic facilities in this part of the world, the incidence of Acanthamoeba keratitis is likely to be reported more often in non-contact lens wearers which may contrast with the decrease in the number of cases reported from developed countries. ${ }^{18}$

The final visual outcome, as measured by resolution of the infiltrate and healing of the ulcer, was achieved in $27 / 31(87.0 \%)$ patients with no significant difference between early and late cases. Use of cationic antiseptics (PHMB and CHx) has resulted in a dramatic improvement in the clinical management of Acanthamoeba keratitis. We used PHMB in $15 / 38(39.4 \%)$ and CHx with PHMB in 23/38 $(60.5 \%)$ patients. Neosporin was used only during sleeping hours in a limited number of patients which may not have played a significant part in control of infection. Although combination therapy with PHMB and propamidine has been reported earlier ${ }^{23}$ the choice of combination therapy with PHMB and $\mathrm{CHx}$ was based on in vitro observation of low MCC (minimum cysticidal concentration) of PHMB and CHx and the reported synergistic activity of both. ${ }^{26}$ The mean duration of treatment in our patients was 106.9 days which is comparable with the duration reported (135 days) for contact lens wearers, ${ }^{18}{ }^{23}$ thereby suggesting that though the clinical features were severe time taken to respond to medical therapy was not unduly long. This also suggests that the susceptibility of Acanthamoeba to biguanides in our series is similar to what has been reported from contact lens wearers.

Studies are taking place at our centre to determine susceptibility of Acanthamoeba isolates from non-contact lens wearers to various antimicrobials and the results (unpublished) support this hypothesis.

Although the visual outcome was poor, a clinical resolution of infiltrate was obtained in majority of the patients with a promise of good vision following optical corneal graft at a later stage. Increased awareness may enable early and frequent recognition and proper management of this devastating corneal disease in patients other than contact lens wearers.

Financial support: Hyderabad Eye Research Foundation, Hyderabad, India.

1 Schaumberg DA, Snow KK, Dana MR. The epidemic of Acanthamoeba keratitis: where do we stand? Cornea 1998;17:3-10.

2 Radford CF, Bacon AS, Dart JKG, et al. Risk factors for Acanthamoeba keratitis in contact lens users: a casecontrol study. BMF 1995;310:1567-70.

3 Kamel AB, Norazah A. First case of Acanthamoeba keratitis in Malaysia. Trans R Soc Trop Med Hyg 1995;89:652. 4 Sharma S, Srinivasan M, George C. Acanthamoeba keratitis
in non-contact lens wearers. Arch Ophthalmol 1990;108: in non-co.

5 Tay-kearney ML, McGhee CN, Crawford GJ, et al. Acanthamoeba keratitis: a masquerade of presentation in six cases. Aust NZ F Ophthalmol 1993;21:237-44

6 Stehr-Green JK, Bailey TM, Visvesvara GS. The epidemiology of Acanthamoeba keratitis in the United States. Am $\mathcal{F}$ Ophthalmol 1989;107:331-6.

7 Stehr-Green JK, Bailey TM, Brandt FH, et al. Acanthamoeba keratitis in soft contact lens wearers. A case control study. $\mathcal{F} A M A$ 1987;258:57-60.

8 Buehler PO, Schein OD, Stamler JF, et al. The increased risk of ulcerative keratitis among disposable soft contact lens users. Arch Ophthalmol 1992;110:1555-8.

9 Auran JD, Starr MB, Jakobiec FA. Acanthamoeba keratitis: a review of the literature. Cornea 1987;6:2-26.

10 Chynn EW, Lopez MA, Pavan-Langston D, et al. Acanthamoeba keratitis. Contact lens and non contact lens thamoeba keratitis. Contact lens and non con
characteristics. Ophthalmology 1995;102:1369-73.

11 Murray PR, Baron EJ, Pfaller MA, et al. Manual of clinical Murray PR, Baron EJ, Pfaller MA, et al. Manual of clinical
microbiology. 6th ed. Washington DC; ASM Press, 1995.

12 Dandona L, Dandona R, Naduvilath TJ, et al. Is current eye-care-policy focus almost exclusively on cataract adequate to deal with blindness in India? Lancet 1998;351: $1312-16$.

13 Whitcher JP, Srinivasan M. Corneal ulceration in the developing world—a silent epidemic. Br $\mathcal{F}$ Ophthalmol 1997;81: 622-3.

14 Srinivasan M, Gonzales CA, George C, et al. Epidemiology and aetiological diagnosis of corneal ulceration in Madurai, South India. Br $\mathcal{F}$ Ophthalmol 1997;81:965-71.

15 Davamani F, Gnanaselvam J, Anandakannan K, et al. Studies on the prevalence of Acanthamoeba keratitis in and around Chennai. Ind f Med Microbiol 1998;16:152-3.

16 Singh S, Sachdeva MPS. Acanthamoeba keratitis. BMf 1994;309:273.

17 Srinivasan M, Channa P, Raju CV, et al. Acanthamoeba eratitis in hard contact lens wearer. Indian 7 Ophthalmol 1993;41:187-8.

18 Radford CF, Lehmann O, Dart JKG. Acanthamoeba keratitis: multicentre survey in England 1992-6. Br f Ophthalmol 1998;82:1387-92.

19 Lindquist TD. Treatment of Acanthamoeba keratitis. Cornea 1998;17:11-16.

20 Pettit DAD, Williamson J, Cabrol GA, et al. In vitro destruction of nerve cell cultures by Acanthamoeba spp. $\mathcal{F}$ parasitol 1996;82:769-77

21 Prajna NV, Pillai MR, Manimegalai TK, et al. Use of traditional eye medicines by corneal ulcer patients presenting to a hospital in South India. Indian $\mathcal{F}$ Ophthalmol 1999; ing to a h:15-18.

22 Niederkorn JY, Ubelaker JE, McCulley JP, et al. Susceptibility of corneas from various animal species to in vitro binding and invasion by Acanthamoeba castellani. Invest Ophthalmol Vis Sci 1992;33:104-12. 
23 Duguid IGM, Dart JKG, Morlet N, et al. Outcome of Acanthamoeba keratitis treated with polyhexamethyl
biguanide and propamidine. Ophthalmology 1997;104: 1587-92.

24 Illingworth CD, Cook SD. Acanthamoeba keratitis. Surv Ophthalmol 1998;42:493-508.
25 Wilhelmus KR, Osato MS, Font RL, et al. Rapid diagnosis of Aacanthamoeba keratitis using calcofluor white. Arch Ophthalmol 1986;104:1309-12.

26 Angel JT, Gabriel MM, Wilson LA, et al. Effect of polyhexamethylene biguanide and chlorhexidine on four species of Acanthamoeba in vitro. Curr Eye Res 1996;15:225-8. 